\title{
Cinq siècles d'histoire religieuse neuchâteloise. Approche d'une tradition protestante, sous la direction de J.-D. Morerod, L. Petris, P.-O. Léchot et F. Noyer
}

\section{Filippo Fassina}

\author{
(2) OpenEdition \\ Journals \\ Edizione digitale \\ URL: http://journals.openedition.org/studifrancesi/6243 \\ DOI: $10.4000 /$ studifrancesi.6243 \\ ISSN: 2421-5856 \\ Editore \\ Rosenberg \& Sellier

\section{Edizione cartacea} \\ Data di pubblicazione: 1 novembre 2010 \\ Paginazione: 541-542 \\ ISSN: 0039-2944

\section{Notizia bibliografica digitale} \\ Filippo Fassina, «Cinq siècles d'histoire religieuse neuchâteloise. Approche d'une tradition protestante, \\ sous la direction de J.-D. Morerod, L. Petris, P.-O. Léchot et F. Noyer», Studi Francesi [Online], 162 (LIV I \\ III) | 2010, online dal 30 novembre 2015, consultato il 09 janvier 2021. URL: http:// \\ journals.openedition.org/studifrancesi/6243 ; DOI: https://doi.org/10.4000/studifrancesi.6243
}

Questo documento è stato generato automaticamente il 9 janvier 2021.

\section{cc) (†)}

Studi Francesi è distribuita con Licenza Creative Commons Attribuzione - Non commerciale - Non opere derivate 4.0 Internazionale. 


\title{
Cinq siècles d'histoire religieuse
} neuchâteloise. Approche d'une tradition protestante, sous la direction de J.-D. Morerod, L. Petris, P.-O. Léchot et F. Noyer

\author{
Filippo Fassina
}

\section{NOTIZIA}

AA. VV., Cinq siècles d'histoire religieuse neuchâteloise. Approche d'une tradition protestante, «Actes du colloque de Neuchâtel» (22-24 avril 2004), sous la direction de J.-D. MOREROD, L. PETRIS, P.-O. LÉCHOT et F. NOYER, Peseux, Université de Neuchâtel, 2009 («Recueil de travaux publiés par la Faculté des lettres et sciences humaines», LV), pp. 412.

1 Segnaliamo qui di seguito i contributi che interessano gli studi cinquecenteschi (II parte).

2 Anne ULLBERG, Les premières chansons imprimés par Pierre de Vingle. La chanson populaire au service de l'Évangile (pp. 41-56): si tratta di un'analisi di tre componimenti di Pierre de Vingle comparsi in tre opuscoli differenti, fra il 1533 e il 1534, con l'intento di divulgare la dottrina protestante alla gente comune. Benché questi testi non presentino un impianto omogeneo e organizzato, è comunque possibile isolare alcuni temi dominanti, fra cui spicca quello della redenzione, anche se non mancano spunti polemici o satirici.

3 Jean DUCHAMP, Les timbres musicaux du premier livre de chansons imprimé par Pierre de Vingle (Neuchâtel, 1533): entre oralité et écriture (pp. 57-74): viene evidenziata l'importanza della musica all'interno dell'opera di Pierre de Vingle, musica che diventa il principale canale di diffusione popolare della dottrina riformata. Vengono presentati diversi tipi di 
chanson e si cerca di ricostruire in modo approfondito le tecniche e i generi musicali che caratterizzavano la rappresentazione di questi componimenti.

William KEMP-Isabelle C. DENOMMÉ, La traduction et l'adaptation de la «Doctrine nouvelle et ancienne» de Rhegius, Genève, 1542-1544; Neuchâtel, vers 1544? (pp. 75-84 e 85-91): la prima parte del contributo (di W. Kemp) si concentra sulla Doctrine nouvelle et ancienne, considerata la traduzione francese della Novae doctrinae ad veterem collatio (1526) del pastore luterano Urbanus Rhegius. Viene infatti ricostruita la complessa storia editoriale di questa traduzione e si instaurano dei confronti testuali per meglio chiarirne il contenuto ideologico e per dimostrare che, con buona probabilità, la revisione generale è stata effettuata da Pierre de Vingle. La seconda parte (di I. C. Denommé) esamina il testo dal punto di vista esclusivamente teologico-dottrinale, evidenziando il carattere riformatore dell'opera. Viene infine affrontata la delicata questione della Messa e dell'Eucarestia, identificando la matrice calvinista che sta alla base di questa traduzione.

5 Geneviève GROSS, Antoine Marcourt. Le «livre des Marchans» et ses rééditions. Miroir d'un conflit entre l'«ancienne» et la «nouvelle» école? (pp. 93-119): in questo studio vengono descritte le varie edizioni del -livre des Marchans, un testo che, sotto la forma di un trattato sul commercio, cela in realtà intenti fortemente anticlericali. Ripercorrendo le varie tappe editoriali $(1533,1534,1541$ e 1544), emergono con grande chiarezza la satira e la polemica contro la Chiesa di Roma e il fine propagandistico a favore delle concezioni calviniste.

6 Michel UMMEL, Les premiers contours d'un anabaptisme neuchâtelois. Autour d'un certain Pierre Pelot (pp. 121-140): l'A. traccia un bilancio della storiografia anabattista, sottolineando la notevole mancanza sia di materiale, sia di storici interni a questo ambiente religioso. Vengono poi elencate alcune delle principali fonti del milieu di Neuchâtel, partendo da una "Confession de foi» distribuita da Pierre Pelot de La Neuveville, che pare abbia dato origine al movimento anabattista in quest'area.

7 Lionel BARTOLINI, Quand Maître Guillaume Farel prêchait au Landeron (pp. 141-147): il presente intervento ha come scopo quello di gettare luce sulla leggenda, secondo la quale il riformatore Guillaume Farel, recatosi a Landeron per predicare, fu cacciato dalle donne del posto, che quasi lo lapidarono. Viene ricostruita la reale presenza del religioso in quell'area, evidenziando, al di là della leggenda, il fatto che effettivamente Landeron rimase un baluardo del cattolicesimo tradizionale contro la dilagante tendenza riformatrice.

8 Jean-François GILMONT, Quelques éditions genevoises de 1539 à 1544 retrouvées dans la bibliothèque de l'Université de Halle-Wittemberg (pp. 149-167): vengono menzionate e brevemente descritte alcune opere stampate a Ginevra fra il 1539 e il 1544 da Jean Girard, opere che risultano preziose per ricostruire il quadro culturale e dottrinale della prima fase della Riforma. Fra gli autori spiccano Olivétan, Rhegius, Farel, Calvino e Melantone, delle cui opere viene riprodotta l'immagine del frontespizio.

William KEMP, Une plaque en émail de 1546 représentant Farel? Considérations sur les présumés portraits de réformateurs par Léonard Limosin (avec une note sur le «Calvin» d'Arcimboldo) (pp. 169-178): l'A. descrive uno smalto di Leonardo Limosino che potrebbe ritrarre il riformatore Guillaume Farel. Tale attribuzione si basa sostanzialmente su un confronto con le Icones de Thèodore de Bèze. Inoltre, viene brevemente presentato un ritratto di Arcimboldo che, secondo alcuni, sarebbe una caricatura di Calvino, volta a sottolineare gli effetti di una eccessiva pratica ascetica, mentre sembra ormai dimostrato che si 
tratti di Johann Ulrich Zasius, vice-cancelliere di Massimiliano I, che fu sfigurato dalla sifilide.

10 Robert WEEDA, Tensions entre Musique et Église à Neuchâtel au temps de Farel (pp. 181-213): vengono descritte dettagliatamente alcune ordonnances promulgate dalle autorità di Berna e finalizzate a regolare minuziosamente la vita religiosa e civile della popolazione. In pieno clima riformato, sono prese di mira la musica e la danza, considerate causa di una gioia incontrollabile e quindi empia. Questa analisi sottolinea la minuziosa casistica dei precetti che, oltre alla musica e alla danza, regolamentano anche le feste e gli strumenti musicali consentiti. 